\title{
Cord Compression from Bony Metastasis: An Important Quality of Life Issue which can be Resolved by a Spinal MDT Tumor Board
}

\author{
Sheikh Muhammad Ebad Ali, ${ }^{*}$, Badaruddin Sahito ${ }^{1}$, Muhammad Amin Chinoy ${ }^{2}$, Ahmed Nadeem \\ Abbasi $^{3}$, Adil Khatri ${ }^{4}$ \\ ${ }^{1}$ Department of Orthopaedics, Dr. Ruth K.M. Pfau, Civil Hospital, Karachi, Pakistan. \\ ${ }^{2}$ Department of Orthopaedics, The Indus Hospital, Karachi, Pakistan. \\ ${ }^{3}$ Department of Oncology, Aga Khan University Hospital, Karachi, Pakistan. \\ ${ }^{4}$ Department of Neurosurgery, Dr. Ruth K.M. Pfau, Civil Hospital, Karachi, Pakistan.
}

Cancer prevalence is increasing over the past few decades. Spinal osseous metastasis is one of the most common sites of secondary neoplastic disease among cancer patients [1]. Spinal malignancies can broadly be classified into primary spinal cancers and secondary spinal metastasis. Metastatic spinal cancers are more common than primary malignancy of the spine. Metastatic spinal cancers are further subdivided into two parts based on the involvement of the dura mater; metastasis external to dura mater can be termed as metastatic epidural spinal cancers (MESC); metastasis inside the dura mater is called metastatic intradural spinal cancers (MISC) [2]. Bony involvements of vertebrae are common at the presentation of MESC while the involvement of the spinal cord and meninges is a prominent feature of MISC. However, the clinical presentation is quite overlapping and timely-decision making is quite challenging in several cases. Several grading systems have been developed to assess the severity of spinal cancers [3-6]. Such ambiguities require a need for teamwork between different specialties to decide the management plan for better patient care. Multidisciplinary Tumor Boards (MDT) are the practical venue where different specialists are present for the discussion [7].

Spinal cancers require teamwork especially between orthopedics, neurosurgery, palliative care, radiation oncology, and medical oncology specialists. But spinal cancers with signs of cord compression are considered an acute emergency where intervention is required within 24-72 hours [8]. Undue delays may lead to irreversible paralysis, sphincter dysfunctions, fecal incontinence, and bladder dysfunctions. Time limitation is the major barrier in reaching a multidisciplinary decision in such cases. Hence, single-handed treatment without a multidisciplinary opinion is a common practice in this particular clinical scenario, which can lead to compromised outcomes.

We hereby, advise a practical solution to this concerning issue. Hospitals are advised to form an on-call team of one or two consultants or senior registrars, from orthopedics, neuro-

*Address correspondence to this author at the Department of Orthopaedics, Dr. Ruth K.M. Pfau, Civil Hospital, Karachi, Pakistan.

Email: sheikh.muhammadebadali14@dmc.duhs.edu.pk surgery, palliative care, radiation oncology, and medical oncology departments who are assigned the task to evaluate patients status of cord compression. coming with spinal neoplasia and reach a conclusive treatment recommendation after multidisciplinary discussion among themselves. The team can be named as Spinal MDT Tumor Board. The team should be accessible when called by the on-duty clinician attending the patient. After reaching a multi-disciplinary recommendation, further workups should be carried out and referral of the patient to the appropriate department should be made. The surgical procedure by neurosurgeons or orthopedic surgeons should be carried out while radiotherapeutic options should be carefully decided by radiation oncologists. It should be made clear that the on-call multidisciplinary team shall only be liable for emergency decision making only and further follow-up and planning of the patient should be carried out in formal regular site-specific MDT Team meetings.

If a pre-designated multidisciplinary team exists in a hospital one can save valuable time and these team members can talk and discuss the case after evaluating the patients' clinical status. If this team is not formed there is a real danger that undue delays that occur while making contacts with specialist teams, may lead to further deterioration of the already manifested neurological deficit.

A quick and early consensus treatment will lead to the reversal of signs and symptoms of paraplegia or quadriplegia. Spinal MDT would prove to be a blessing for neurologically deteriorating patients who genuinely need a swift treatment decision by specialists belonging to various disciplines.

\section{CONFLICT OF INTEREST}

Declared none.

\section{ACKNOWLEDGEMENTS}

Declared none. 


\section{REFERENCES}

[1] Hong S, Youk T, Lee SJ, Kim KM, Vajdic CM. Bone metastasis and skeletal-related events in patients with solid cancer: A Korean nationwide health insurance database study. PLoS One 2020; 15(7): e0234927. DOI: 10.1371/journal.pone.0234927

[2] Schick U, Marquardt G, Lorenz R. Intradural and extradural spinal metastases. Neurosurg Rev 2001; 24(1): 1-5. DOI: 10.1007/PL00011959

[3] De la Ramos RG, Benton JA, Gelfand Y, et al. A novel clinical scoring system for perioperative morbidity in metastatic spinal tumor surgery: The spine oncology morbidity assessment score. Spine (Phila Pa 1976) 2021; 46(3): E161-E6. DOI: 10.1097/BRS.0000000000003733

[4] He X, Jiao Y-Q, Yang X-G, Hu Y-C. A novel prediction tool for overall survival of patients living with spinal metastatic disease. World Neurosurg 2020; 144: e824-e36. DOI: 10.1016/j.wneu.2020.09.081

[5] Uei H, Tokuhashi Y, Maseda M. Relationship between paralysis and the epidural spinal cord compression scale in spinal metastasis. Orthopedics 2020; 43(6): e567-e73. DOI: 10.3928/01477447-20200812-09

[6] Dakson A, Leck E, Brandman DM, Christie SD. The clinical utility of the Spinal Instability Neoplastic Score (SINS) system in spinal epidural metastases: A retrospective study. Spinal Cord 2020; 58(8): 892-9. DOI: 10.1038/s41393-020-0432-8

[7] Abbasi AN. Cancer management is a multidisciplinary team work. J Coll Physicians Surg Pak 2011; 21(5): 259-61.

[8] Frank AR. Spinal cord compression--an oncologic emergency. Nebr Med J 1990; 75(8): 230-5. 